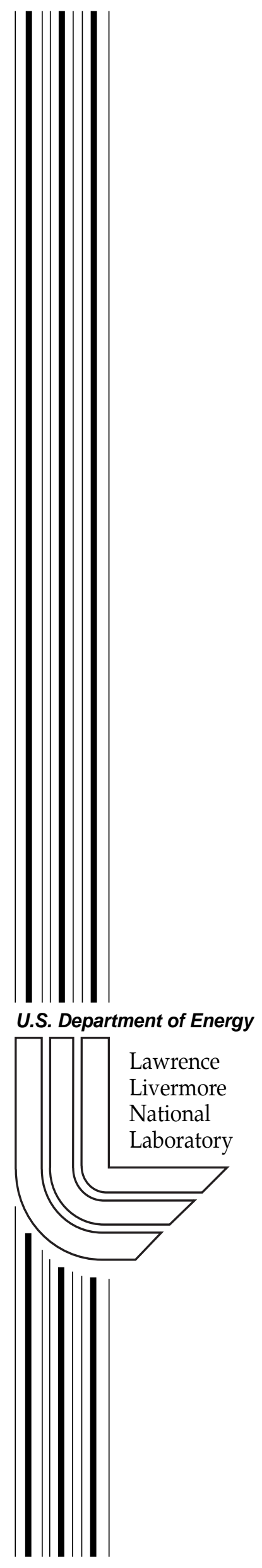

UCRL-ID-153417

\title{
Analysis of Shock-Void Experiment (U)
}

D.T. Woods, H. Robey, P. Stry

May 2, 2003 


\section{DISCLAIMER}

This document was prepared as an account of work sponsored by an agency of the United States Government. Neither the United States Government nor the University of California nor any of their employees, makes any warranty, express or implied, or assumes any legal liability or responsibility for the accuracy, completeness, or usefulness of any information, apparatus, product, or process disclosed, or represents that its use would not infringe privately owned rights. Reference herein to any specific commercial product, process, or service by trade name, trademark, manufacturer, or otherwise, does not necessarily constitute or imply its endorsement, recommendation, or favoring by the United States Government or the University of California. The views and opinions of authors expressed herein do not necessarily state or reflect those of the United States Government or the University of California, and shall not be used for advertising or product endorsement purposes.

This work was performed under the auspices of the U. S. Department of Energy by the University of California, Lawrence Livermore National Laboratory under Contract No. W-7405-Eng-48.

This report has been reproduced directly from the best available copy.

Available electronically at http://www.doc.gov/bridge

Available for a processing fee to U.S. Department of Energy

And its contractors in paper from

U.S. Department of Energy

Office of Scientific and Technical Information

P.O. Box 62

Oak Ridge, TN 37831-0062

Telephone: (865) 576-8401

Facsimile: (865) 576-5728

E-mail: reports@adonis.osti.gov

Available for the sale to the public from

U.S. Department of Commerce

National Technical Information Service

5285 Port Royal Road

Springfield, VA 22161

Telephone: (800) 553-6847

Facsimile: (703) 605-6900

E-mail: orders@ntis.fedworld.gov

Online ordering: http://www.ntis.gov/ordering.htm

\section{OR}

Lawrence Livermore National Laboratory

Technical Information Department's Digital Library

http://www.llnl.gov/tid/Library.html 


\title{
Analysis of Shock-Void Experiment(U)
}

\author{
D. Tod Woods ${ }^{1}$, Harry Robey ${ }^{2}$, and Pete Stry ${ }^{3}$
}

May 2, 2003

1. Lawrence Livermore National Laboratory, Univ. of California L-16, P.O. Box 808, Livermore, CA. 94550. e-mail: tod@eaglevail.llnl.gov

2. Lawrence Livermore National Laboratory, Univ. of California L-21, P.O. Box 808, Livermore, CA. 94550. e-mail: robey1@llnl.gov

3. Lawrence Livermore National Laboratory, Univ. of California L-22, P.O. Box 808, Livermore, CA. 94550. e-mail: stry1@llnl.gov

This work was performed under the auspices of the U.S. Department of Energy by the University of California Lawrence Livermore National Laboratory under contract No. W-7405Eng-48. 


\begin{abstract}
We compare CALE simulations with recent experimental results of a laser-induced shock traversing a spherical under-dense region ("void"). In this report the experimental results are described, as well as several numerical attempts at explaining the observed radiographs. Our conclusion is that the numerical simulations at this time cannot satisfactorily explain the experiment. The simulations also indicate that the introduction of air gaps between the under-dense sphere and the surrounding foam can greatly change the behavior of the shocked sphere. Thus fabrication details may play an important role in the detailed evolution of this experiment. Regardless of the simulations, analysis of the observed time sequence indicates that reproducibility of this experiment may be a factor. To settle this issue, further experiments of this kind will be required.
\end{abstract}




\section{Introduction}

We compare recent experimental results of a laser-induced shock traversing a spherical underdense region ("void") with CALE simulations of the event. The importance of this experiment is to help ascertain the effect of shock propagation through under-dense regions which may exist due to imperfections in the fabrication process. As such, a target sample is constructed with a rarefied spherical ball which is doped with Iodine to make it opaque at the energy of the backlighter. We describe the details of the setup in section 2. The results from the experiment are summarized in section 3. Our attempts at reproducing the experiment using CALE simulations is described in section 4. Finally, our conclusions are given in section 5 .

\section{Problem Setup}

The basic setup for the experiment is summarized in Figure 1. This shows a cross-section of the cylindrical tube sample with the radial coordinate as the ordinate, and the z-coordinate,

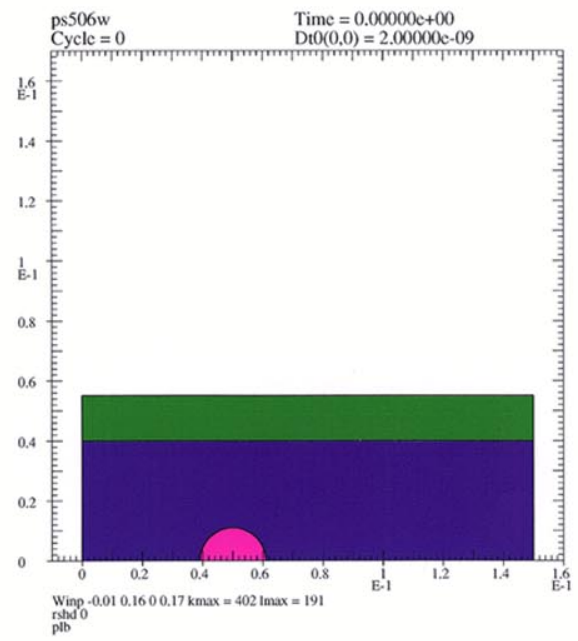

Figure 1: Initial configuration of the cylindrical target. Green is Be tube. Blue is polystyrene foam. Magenta is the underdense Iodo-Resorcinol Formaldehyde foam. along the tube, as the abscissa. There are three components to the target sample. (1) First is the Polystyrene $(\mathrm{CH})$ foam which is shaded blue. It has a mass density of $\rho_{\mathrm{CH}}=1.05 \mathrm{~g} / \mathrm{cm}^{3}$. The tube has a total length of $0.15 \mathrm{~cm}$, and the radius of the foam is $0.04 \mathrm{~cm}$. (2) The second component is a sphere (shaded magenta in Fig. 1) of Iodo-Resorcinol Formaldehyde (IRF) foam-a new material produced by Joe Satcher (CCHED). It is a plastic doped with Iodine to make it opaque at the backlighter energy of $6.7 \mathrm{keV}$. The sphere is placed on axis at a distance of $0.05 \mathrm{~cm}$ from the front surface, with a radius of $0.011 \mathrm{~cm}$. The density of the sphere $\rho_{I R F}=0.5 \mathrm{~g} / \mathrm{cm}^{3}$, roughly half of the surrounding polystyrene. The empirically determined chemical formula for the Iodine-doped plastic is approximately $\mathrm{C}_{21} \mathrm{H}_{19} \mathrm{O}_{9} \mathrm{I}_{2}$. (3) The third region is the 
Beryllium tube which surrounds the plastic (shaded green in Fig. 1). It extends the entire length of the tube $(0.15 \mathrm{~cm})$ with an annulus extending from $R=0.04 \mathrm{~cm}$ to $0.055 \mathrm{~cm}$. The density of the annular shell is $\rho_{B e}=1.845 \mathrm{~g} / \mathrm{cm}^{3}$.

The sample is then illuminated from the left by the laser. The laser is on for a time $t_{\text {laser }}=1 \mathrm{~ns}$, which ends up depositing an energy of $E_{\text {laser }}=2.5 \mathrm{KJ}$. The laser deposition is modeled as a simple square pulse over the duration $t_{\text {laser }}$.

\section{Experimental Results}

The experimental results were obtained by Harry Robey on a shot on Omega in August, 2002. The experimental results are shown in Figures 2-5 at the times $t=10,20,30,40$ s. On each slide is the side-on radiograph and a face-on radiograph (although CCD errors prevented face-on pictures at $t=20$ and $t=30 \mathrm{~ns}$ ). Also shown for comparison on each slide is the simulated radiograph from earlier CALE calculations of Pete Stry. The lower half of the simulated radiograph figure simply shows the location of the material interface between the sphere and Polystyrene. The calculations presented later are considered to supersede these earlier calculations, as we will discuss.

Figure 2 shows the experimental results at $t=10 \mathrm{~ns}$. We see from the side-on radiograph that the shock is roughly halfway through the sphere. We note that the shock appears perfectly planar, and its location inside the sphere appears to be the same as that of the shock exterior to the sphere. That is, it appears from the experimental radiograph that the shock is running at the same speed inside the sphere, as it is outside the sphere. This certainly seems counter-intuitive, and we discuss it more in the next section. Also shown at $t=10 \mathrm{~ns}$ is the face-on radiograph. At this point we can discern nothing unusual about this image. One can get a handle for the "graininess" of the radiographs from these images, since at this point we believe the samples to be uniform, and the hydrodynamics insufficient to have produced any noticeable breakup of the uniformity.

At $t=20 \mathrm{~ns}$ (Figure 3 ) the shock should be through the sphere, emerging through the other side. The side-on radiograph at this time is quite remarkable. The sphere is now fully compressed, and highly elongated. Unfortunately, it appears the radiograph is not centered on the sphere, thus leaving out the upper part of the sphere. With some ambiguity, the center of the compressed sphere can be located in the upper part, with two dark rollups at about the original radius of the sphere. Extending down from the lower rollup appears to be more material from the sphere in a highly mixed state. The overall shock is difficult to make out in this picture. However, one guess puts it parallel to the $200 \mu \mathrm{m}$ line. If this is true, then the results are difficult to understand since it would appear that the axis of symmetry in the sphere is now at an angle to the shock normal. But given the "graininess" of the picture the precise location of the shock is uncertain. Overall, the essential characteristics are the main body of the sphere is compressed, and the outer edges appear to be undergoing hydrodynamic instabilities and expanding radially (i.e. up and down in this orientation). There is no face-on radiograph at this time. 


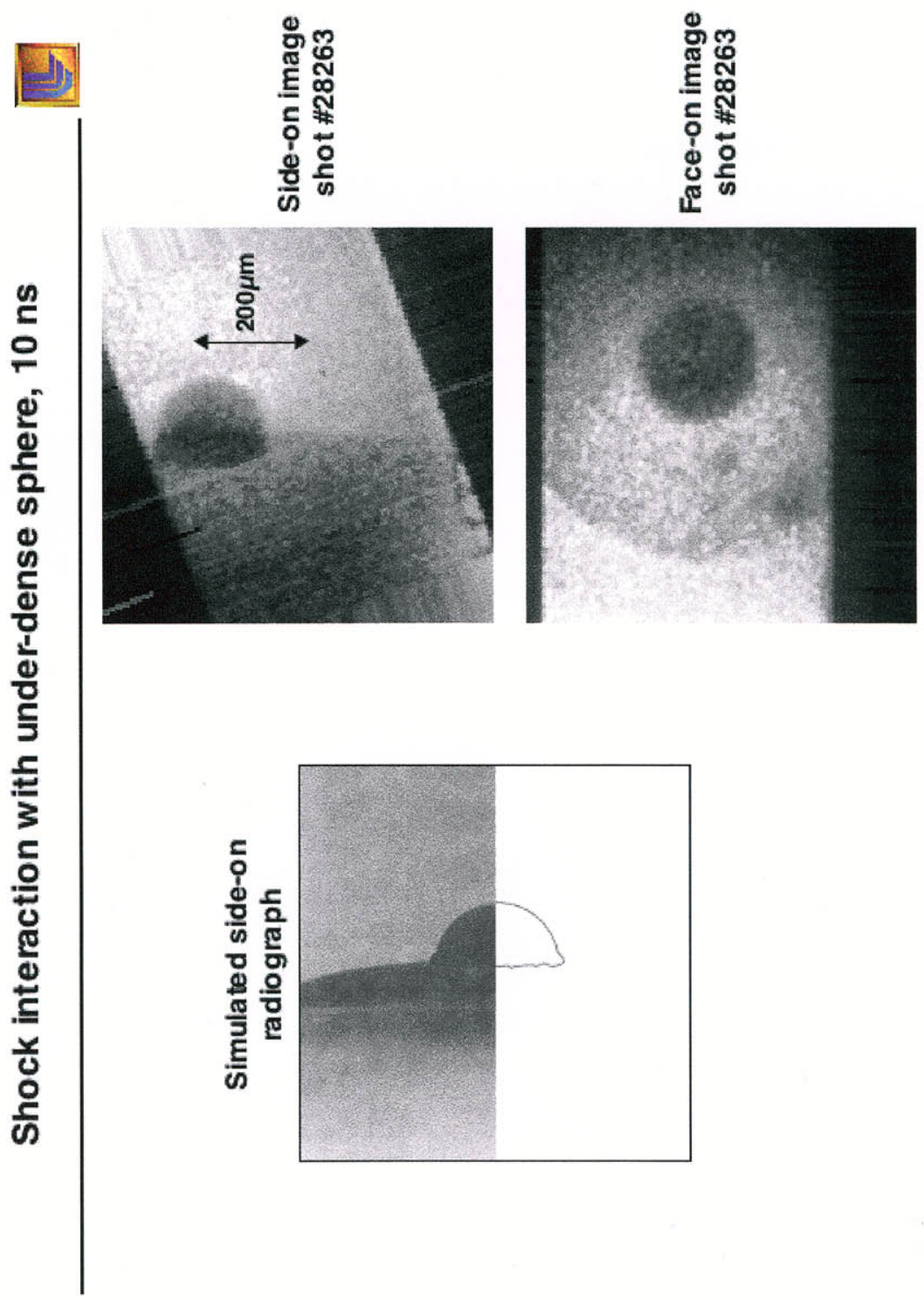

Figure 2: $t=10 \mathrm{~ns}$. 

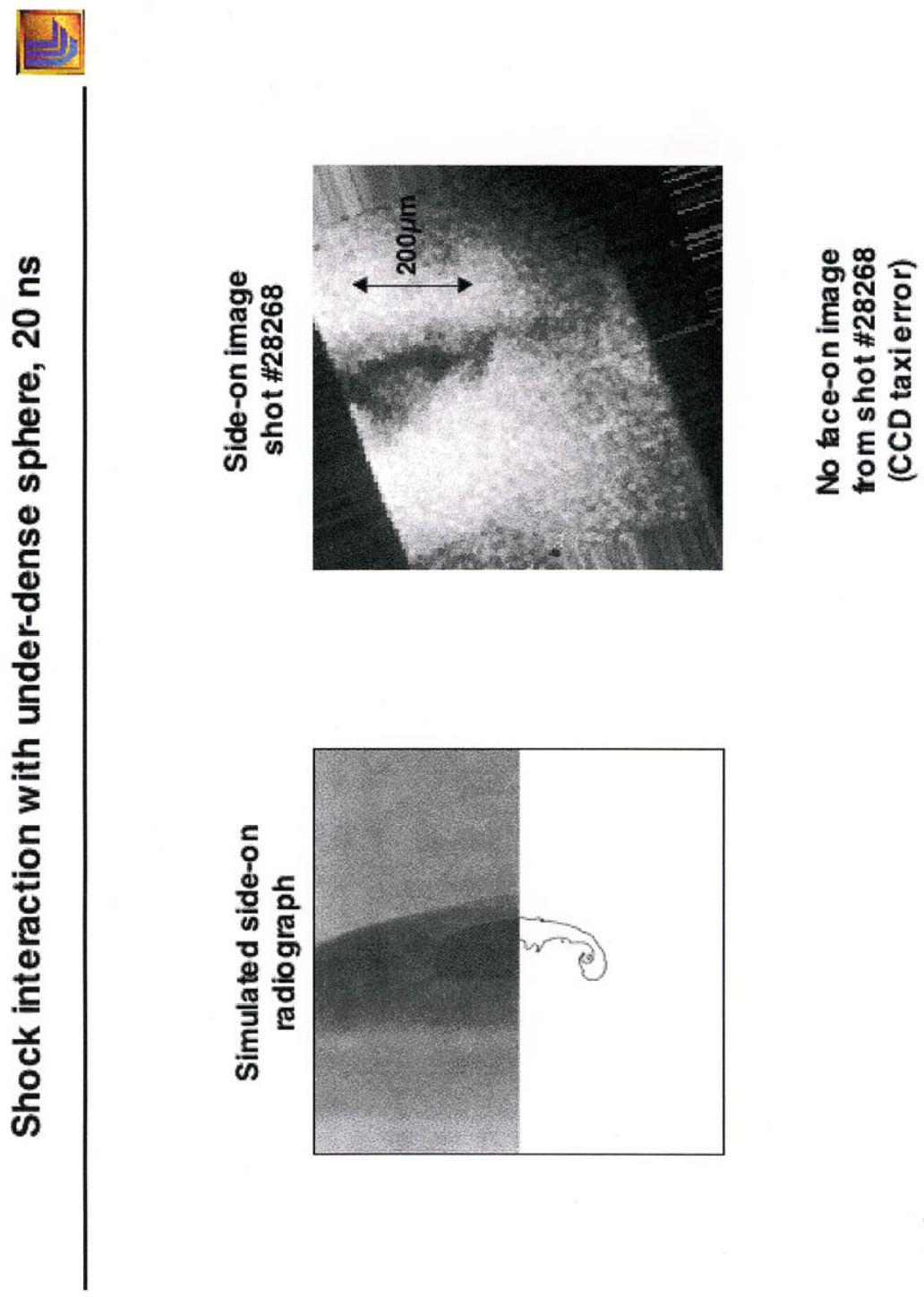

Figure 3: $t=20 \mathrm{~ns}$. 
The results at $t=30 \mathrm{~ns}$ are shown in Figure 4. Again we only have a side-on radiograph at this time. There appear to be rollups at top and bottom of the sphere, which are now ahead of the main body of the sphere. This is an unexpected result and difficult to explain with simulations, as we will see in the next section. Notice also the shock is more clearly defined in this radiograph. It now appears definitively that the axis of symmetry in the sphere is at an angle to the normal of the shock. However, we admit that an axis of symmetry is somewhat difficult to define- which in and of itself is a curious result. We have no explanation for perceived angle between the shock and the axis of symmetry of the sphere. The overall thickness of the sphere appears to have greatly increased. If the simulations are a guide, this may be a projection effect. That is, it may be that only the outer edges of the sphere are experiencing rollups, and gradually falling behind. These features when projected in revolution give the appearance that even the central part of the sphere has expanded out in the $Z$-direction. But this may not be the case, as evidenced by the simulated radiograph which shows the central part of the sphere is still quite thin.

Our last image is at $t=40 \mathrm{~ns}$ (Figure 5). This image seems the most remarkable of the set. First of all notice the shock well past the sphere, as it should be. Only now the shock normal appears aligned with what appears now to be a well defined axis of symmetry of the sphere. It is unclear how the symmetry of the sphere could improve between $t=30 \mathrm{~ns}$ and $t=40 \mathrm{~ns}$-let alone how it could realign itself with the shock normal. Notice also that the rollups which were leading the main body of the sphere have now disappeared. Not only do we have no explanation for how the rollups could lead the main body of the sphere, we further have no explanation for how they could disappear. The main body of the sphere now has a nice clear curvature to the leading edge; something which could not be see at all at $t=30 \mathrm{~ns}$. There does appear to be more material at large radii which may have broken off from the main sphere. Overall, this snapshot will show appreciable agreement with the simulations. However, it is difficult to understand how this image follows from the observation at $t=30 \mathrm{~ns}$. The image at $t=30 \mathrm{~ns}$ is one in which rollup material at large radii leads the main body of the sphere. The leading edge of the sphere is nearly planar, with an approximately hemispherical trailing edge. While at $t=40 \mathrm{~ns}$, there is no evidence of rollup material leading the sphere. The leading edge of the compressed sphere is now curved, and the trailing edge is now nearly planar. Overall these two pictures seem completely at odds with each other. We believe there may be issues of reproducibility in this experiment. Because of insufficient number of beams we could afford the backlighter at only one time per shot. Therefore our "sequence" of snapshots are the result of completely different shots. The most logical explanation appears to be that each of these shots were not as "identical" as we would of like. We can only settle this through more shots at the same times as our current experiment. Only by viewing the constancy of several experiments at the same time can we determine if what we are seeing is real. However, for now it seems reasonable to question the reproducibility of the experiment. 

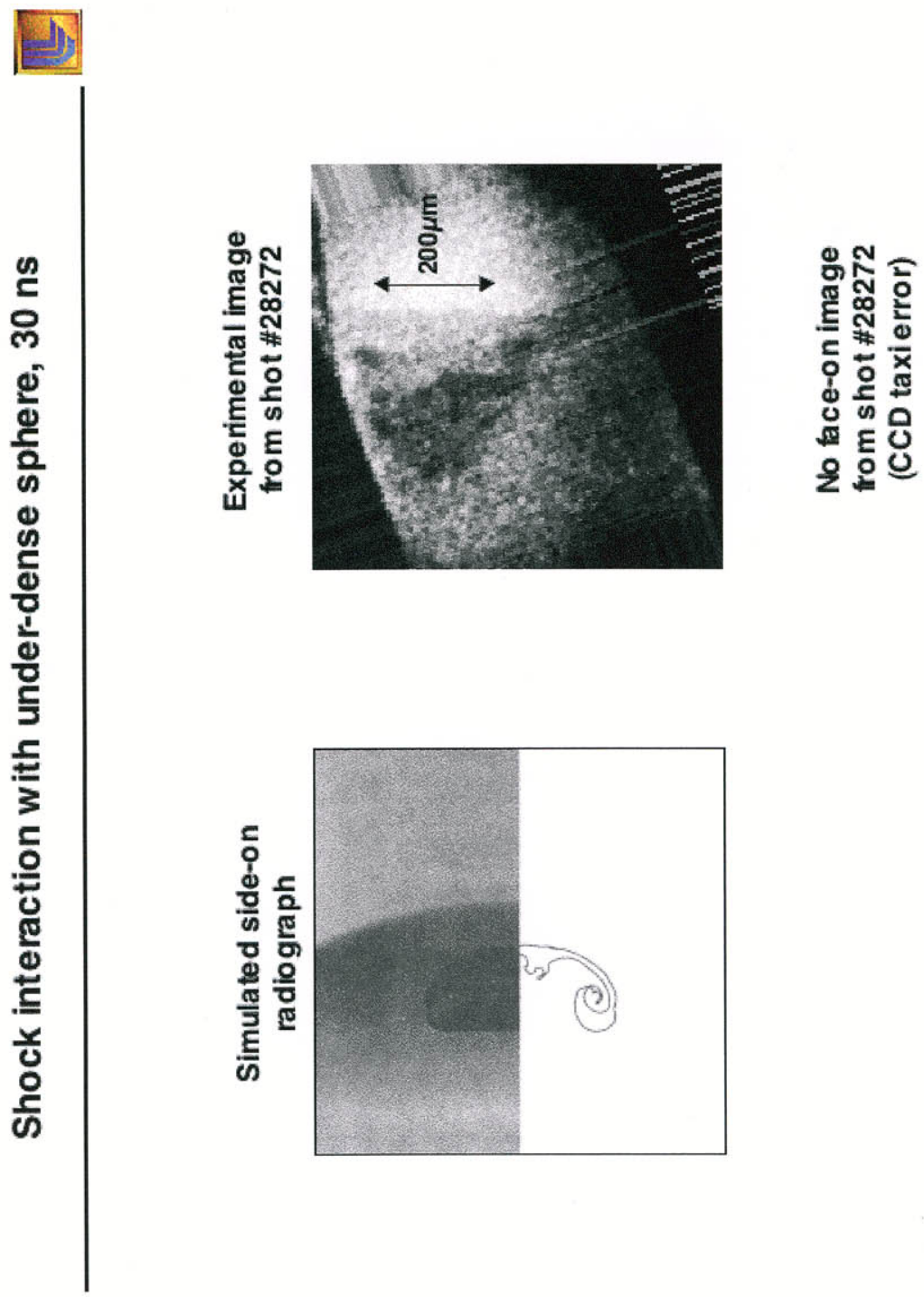

Figure 4: $t=30 \mathrm{~ns}$. 


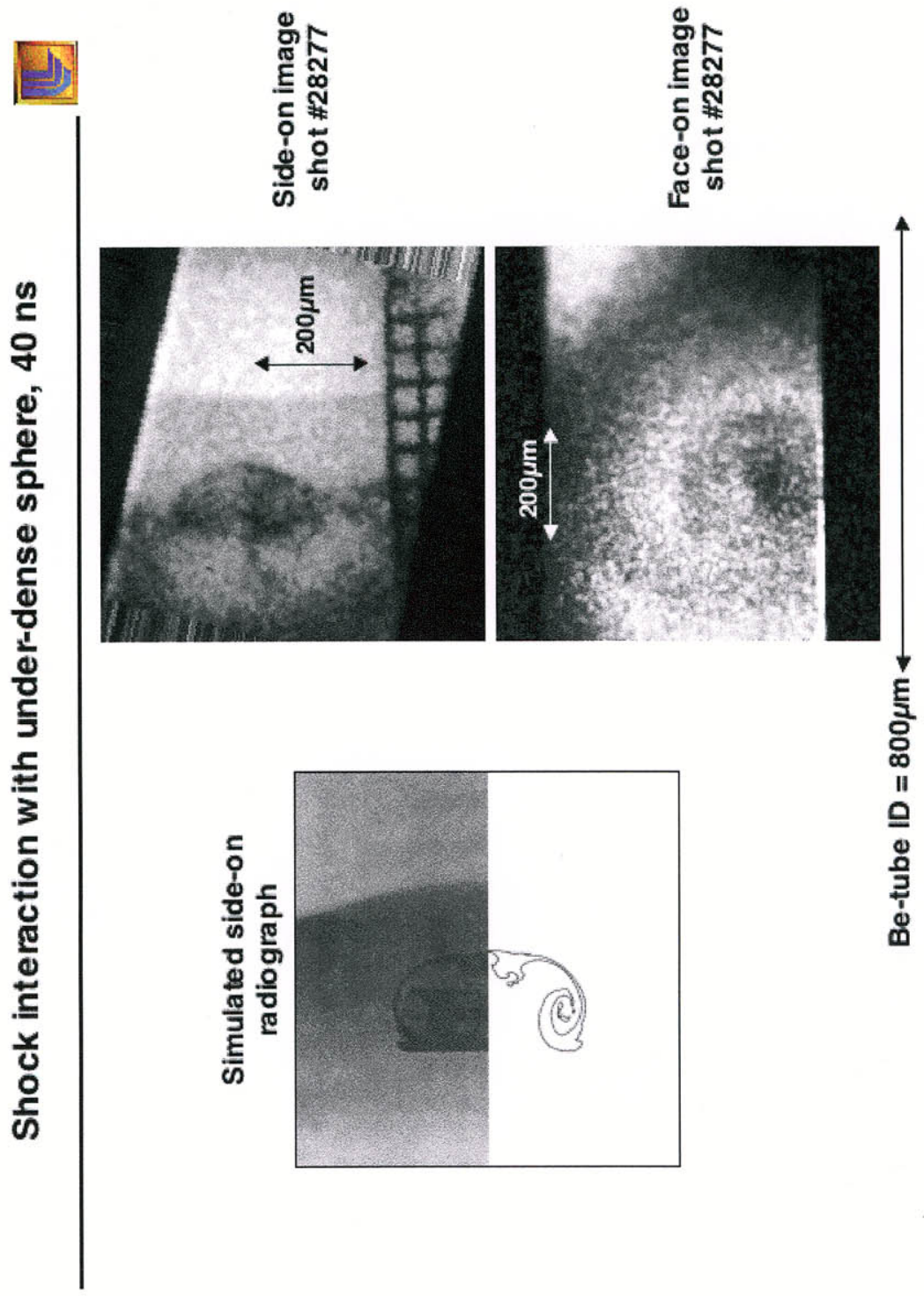

Figure 5: $t=40 \mathrm{~ns}$. 


\section{CALE Simulations}

\subsection{Standard Model}

We describe in this section the setup in CALE of our "standard" model and the results. We also describe some of the variation in the parameters investigated.

\subsubsection{Model Parameters}

The zoning consists of $402 \mathrm{k}$-lines and 191 l-lines so that there are 401 zones in the Z-direction and 190 zones in the R-direction. The zone size is roughly $2.5 \mu$, with some slight deviations. For example, the zone size is ratioed in the Z-direction by $0.5 \%$ per zone from the left face (where the laser hits) until $z=0.03 \mathrm{~cm}$. At $z=0$ the zone size in the $z$-direction is roughly $1.0 \mu$, ramping up to $2.5 \mu$ at $z=0.03$. There is constant zoning in the z-direction from $z=0.03 \mathrm{~cm}$ out to $z=0.07 \mathrm{~cm}$. From $z=0.07 \mathrm{~cm}$ out to $z=0.15 \mathrm{~cm}$ the zone size in the Z-direction increases quickly ( $5 \%$ per zone) from $2.5 \mu$ to $\approx 40 \mu$ at the back edge. In the $\mathrm{R}$-direction, the zoning is uniform at $2.5 \mu$ out to the Be-tube at $R=0.04 \mathrm{~cm}$. Inside the Be-tube the zoning increases in the radial direction at a $5 \%$ rate per zone, thus increasing the size from $2.5 \mu$ out to $10 \mu$ at the edge. These is of course just the initial zoning. The calculations are run in ALE mode.

Calculations include the effects of thermal conduction and radiative diffusion. Initially, Lagrange subcycling is turned on (set to 5), meaning there will be 5 hydro subcycling before an full energy calculation.

The laser deposition is handled by sourcing in a "ray disc" card. There are nray $=8000$ rays parallel to the $\mathrm{Z}$-axis at a distant location $(z=-10 \mathrm{~cm})$. The power for each ray is assumed to be uniform in the R-direction and extends out to the radius of the plastic at $R=0.04 \mathrm{~cm}$. The critical electron density is set at $n_{\text {crit }}=9.1 \times 10^{21} \mathrm{~cm}^{-3}$, which determines where the laser energy is dumped. Finally, we have set the parameter which controls the "speckling" of the laser, lrayscat $=1 \mu$. This parameter controls the angle of the rays in that it sets up a gaussian distribution in time for a given ray with a half-width of lrayscat impinging on the sample. A simple square wave is used to model the energy deposition with time. The laser is turned on at $t>0$ for $1 \mathrm{~ns}$, at which time a total of $2.5 \mathrm{KJ}$ is deposited in the sample.

The boundary conditions are basically "free" surfaces, except the symmetry plane at $R=0$, where the radial velocity is set to zero. For radiation diffusion, the front and outer radius are treated as open boundaries, so that a Milne type boundary condition is applied.

Standard tables were used for the EOS of the Be and the Polystyrene. A special EOS table was constructed by David Young (PAT) for the Iodo-Resorcinol foam. The opacity for the three materials at the energy of the Fe backlighter $(6.7 \mathrm{KeV})$ are $\kappa_{B e}=1.8, \kappa_{C H}=7.4$, and $\kappa_{I R F}=181 \mathrm{~cm}^{2} / \mathrm{g}$, thus indicating the IRF is nearly 25 times as opaque (per gram) as the $\mathrm{CH}$. 


\subsubsection{Run-time Details - Some of the Warts}

As with most codes, sometimes alterations to the standard physics is necessary to promote stability. For our problem, the primary source of difficulty is in the low density blow-off region. The zones become distorted due to large velocities. Also, the blow-off region can wrap around the sample, actually leading the shock outside of the Be annulus. This ends up producing an acute angle such that the rezoning from the ALE has difficulty in producing acceptable zoning. To keep this from happening, a few things are done to promote stability. These alterations were based upon the experience of Pete Stry during his running of earlier simulations for this problem. First of all, at $t=1 \mathrm{~ns}$ when the laser turns off, the variable $r d f l x$ is set to 0.9 . This is important in low optical depth, distorted (elongated) zones. Further stability is needed at $t=1.5 \mathrm{~ns}$ when $r d f l x$ is lowered further to 0.8 , Lagrange subcycling is turned off ( $n d t g x=1)$, and in just the blow-off region the Planck mean opacity is increased by the factor $10^{4}$ to prevent the electron and ion temperatures from getting out of equilibrium in very low density zones. The blow-off region is defined by the k-lines 1 and 184, where $z(k=184)=0.03 \mathrm{~cm}$, initially. This is the ablative region where the zone size in the $z$-direction increases from $1.0 \mu$ at the left edge $(z=0)$ to $2.5 \mu$ at $z=0.03 \mathrm{~cm}$. Finally, to prevent the acute angle from developing as the blow-off comes out and around the tube, the radial velocity is set to zero in the blow-off region, as in ablation of a planar wall.. We find that limiting the radial expansion of the blow-off region only during the laser deposition, i.e. out to $t=1 \mathrm{~ns}$, is sufficient to prevent acute angles from occurring due to the blow-off. We readily admit that this invalidates the behavior of the blow-off in detail, however, since our primary focus is on how the shock interacts with the low-density sphere embedded in our sample, this restriction is acceptable.

We have found the ad-hoc procedure of zeroing out the radial velocity in the blow-off region to have an unexpected behavior in the post-shock flow. To illustrate this, we show the density at $t \approx 5 \mathrm{~ns}$ from two calculations in Figure 6 . The two calculations differ only in how long the radial velocity in the blow-off region is set to zero. The first calculation sets the radial velocity to zero in the blow-off region for all time. Notice striations in the post-shock region-alternating bands of overdense and underdense regions. Similar behavior is seen in the temperature and also the pressure, although to a lesser degree. That is, where the density is low, the temperature is high, and the pressure is slightly too low. In the second calculation the radial velocity is set to zero in the blow-off region only while the laser is on, i.e. up to $t=1 \mathrm{~ns}$. The result is that at $5 \mathrm{~ns}$ the post-shock flow is now nice and uniform. Thus it appears, our decision to limit the radial velocity in the blow-off region has greatly impacted the energy, and to some small extent the momentum equations. Consequently, we advise against this procedure once the laser deposition has ceased. Note also in Figure $6 \mathrm{~b}$ how the blow-off has come around the Be-tube, producing an acute angle in the outer radial boundary. It is this feature which can give us the most difficulty in keeping the computation stable. 

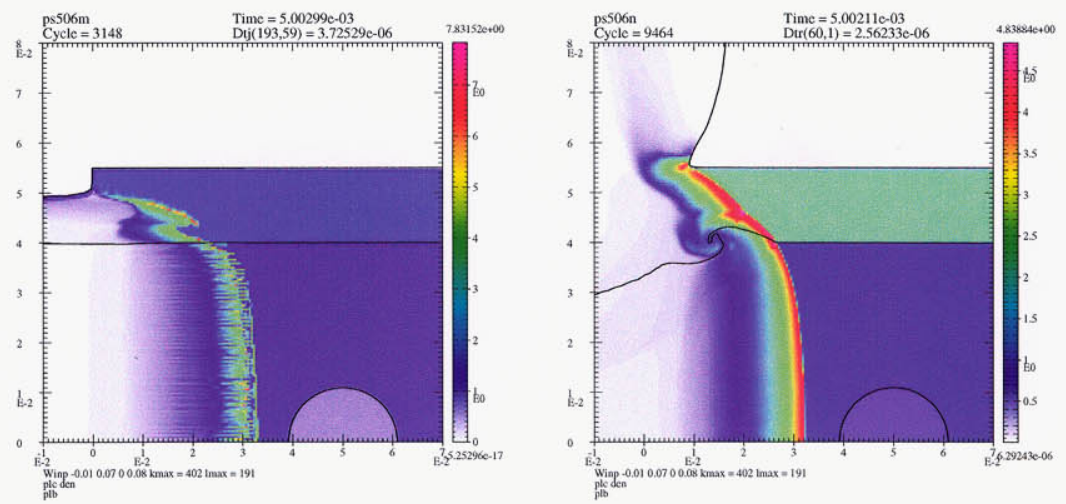

Figure 6: Density at $t=5 \mathrm{~ns}$. First figure shows results when radial velocity set to zero in blowoff region for all time produces density striations. Second figure has radial velocity set to zero in blow-off region only during laser deposition $(t=1 \mathrm{~ns})$-no striations.

\subsubsection{Results}

We now discuss the results from our "standard" CALE simulation. The density contours are shown for six different times: $t=1,5,10,20,30,40 \mathrm{~ns}$ in Figures 7-9. The last four times correspond with the times of the experimental radiographs. At $t=1 \mathrm{~ns}$ the laser turns off. We see that the shock front is nearly planar. There is a slight curvature near the outer edge. Our model setup does not provide for direct irradiation of the Be. The heating and subsequent blow-off in the $\mathrm{Be}$ is due to thermal conduction and radiation diffusion (mostly conduction). There is also a slight density enhancement near the symmetry axis near $R \approx 0$. At this point we have no explanation for this feature. Notice that there is no blow-off in the radial direction since $v_{R}$ was still being set to zero during laser deposition. The laser turns off at $t=1 \mathrm{~ns}$ and the shock starts propagating down the tube. At $t=5 \mathrm{~ns}$ the shock front is around $Z=0.03 \mathrm{~cm}$. At this point the shock, out to roughly the radius of the sphere, is nearly planar. There is no evidence of the enhanced density near $R=0$ seen at $t=1 \mathrm{~ns}$. The curvature of the shock at large radii is increasing, turning the shock to an angle of nearly 45 degrees in the Be. Notice early on that the shock is strong and the $\mathrm{CH}$ is behaving roughly as a $\gamma=5 / 3$ gas with the density behind the shock at roughly $4 \mathrm{~g} / \mathrm{cm}^{3}$. At $t=10 \mathrm{~ns}$ the shock has hit the sphere. The shock-front just outside the sphere is near $Z=0.05 \mathrm{~cm}$, i.e. near the center of the sphere. Notice that inside the sphere the shock is running slightly ahead-the leading edge being around $Z=0.054 \mathrm{~cm}$. This is slightly different from the observed radiograph where it appears that the shock front is basically planar, even inside the sphere. Based upon simple hydrodynamics you would expect the shock to speed up slightly inside the more rarefied sphere. It is difficult to explain the experimental result 

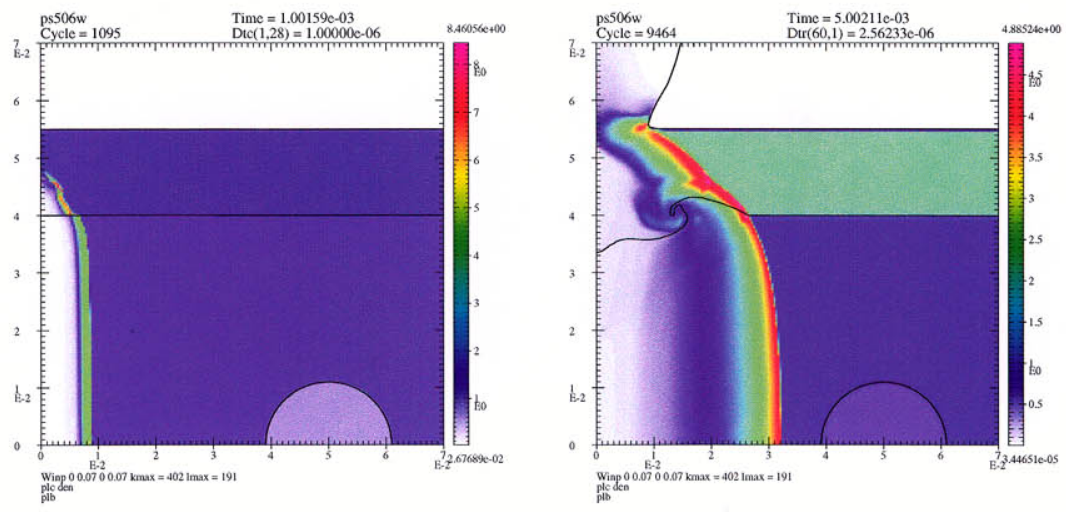

Figure 7: First figure shows the density at $t=1 \mathrm{~ns}$, i.e. when the laser turns off. Second figure shows the density at $t=5 \mathrm{~ns}$. Shock front is still basically planar, although there is some curvature of the shock in the $\mathrm{CH}$ near the Be-tube.
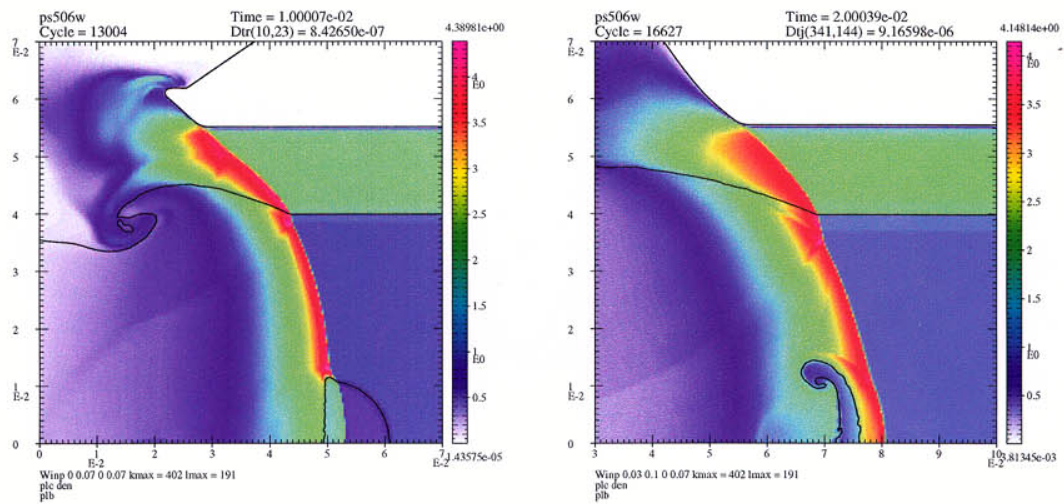

Figure 8: First figure shows the density at $t=10 \mathrm{~ns}$. The shock is roughly halfway through the sphere. Notice that the shock inside the sphere runs ahead of that outside the sphere. Second figure shows the density at $t=20 \mathrm{~ns}$. Shock front has progressed through the sphere and compressed it up. There is a rollup of the outer parts. 

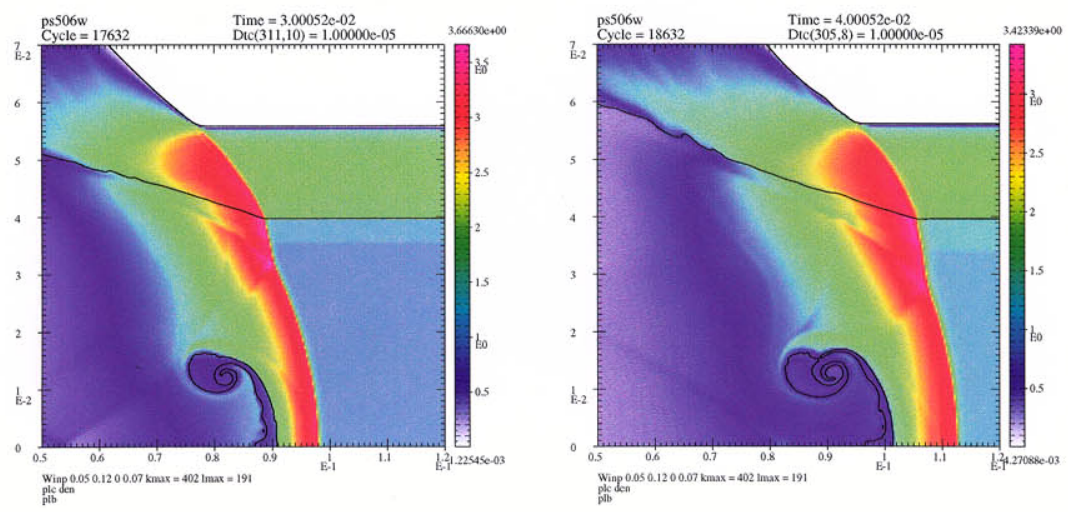

Figure 9: First figure shows the density at $t=30 \mathrm{~ns}$. Second figure shows the density at $t=40 \mathrm{~ns}$.

that no such acceleration of the shock front is observed. Simple calculations would suggest that a roughly $15 \%$ increase in the shock speed should be expected when the shock enters the sphere when there is a density drop to roughly half that of the ambient medium. This behavior is exhibited clearly in the CALE simulations, but no evidence is indicated in the experiment. At $t=20 \mathrm{~ns}$ the shock has emerged from the sphere. The sphere is now fully compressed and moving along at the post-shock speed at roughly $1.5-2.0 \mathrm{~cm} / \mu \mathrm{sec}$. There is a well-defined rollup at the top of the sphere due to Richtmeyer-Meshkov and Kelvin-Helmholtz instabilities. The outer edge of the sphere has expanded radially from $R=0.011 \mathrm{~cm}$ to roughly $R=0.015 \mathrm{~cm}$. Figure 9 a shows the density at $t=30 \mathrm{~ns}$. The main body of the sphere remains compressed and advected downstream to $Z=0.09 \mathrm{~cm}$. The vortex behavior in the outer sphere has continued, producing a very nice, well-defined rollup. The shock is weakening as evidenced by the post-shock densities in the $\mathrm{CH}$ being now only $\approx 3 \mathrm{~g} / \mathrm{cm}^{3}$. The outer edge of the sphere is expanded further radially, now extending out to $R=0.017 \mathrm{~cm}$. Finally, in Figure $9 \mathrm{~b}$ the density is shown at $t=40 \mathrm{~ns}$, the latest time for which we have experimental data. The leading edge of the sphere has advected to $Z=0.101 \mathrm{~cm}$. The rollup in the outer part of the sphere has continued. This motion has caused a thinning, which in later time will lead to breakup into individual pockets of material. The rollup leads to a lengthening between the leading and trailing edges, roughly a distance of $0.02 \mathrm{~cm}$ in the Z-direction, compared to $\approx 0.01 \mathrm{~cm}$ at $t=20 \mathrm{~ns}$. Also note that the overall shock front appears to be straightening.

In Figure 10 we show the material temperature at two times $t=20 \mathrm{~ns}$ and $t=40 \mathrm{~ns}$. In both cases the hottest material is the shocked up sphere, being roughly $10 \mathrm{eV}$ at $t=20 \mathrm{~ns}$ and $6 \mathrm{eV}$ at $t=40 \mathrm{~ns}$. Thermal conduction provides a loss mechanism out of the shocked clouds, which reduces pressure support inside the cloud, resulting in a narrowing or thinning of the material boundary at $t=40 \mathrm{~ns}$. 

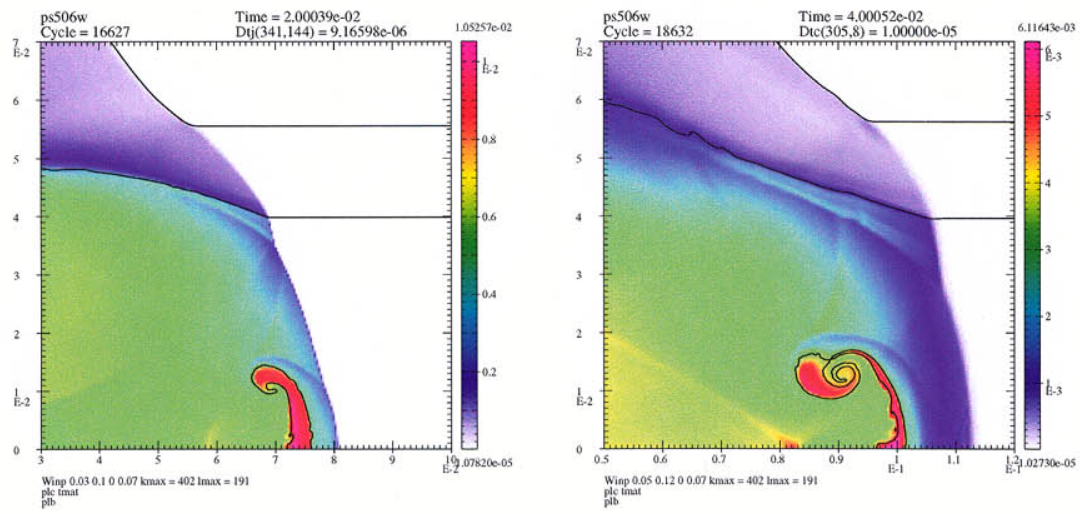

Figure 10: First figure shows the temperature at $t=20 \mathrm{~ns}$. The material inside the sphere has been shocked up to about $10 \mathrm{eV}$. The post-shock $\mathrm{CH}$ is roughly $3-4 \mathrm{eV}$. Second figure shows the temperature at $t=40 \mathrm{~ns}$. The material inside the sphere has now cooled to roughly $5-6 \mathrm{eV}$, and the shock is heating the $\mathrm{CH}$ up to only roughly $1 \mathrm{eV}$.

In Figure 11 we show the pressure as a function of Z-coordinate at a height $R=0.02 \mathrm{~cm}$ for different times. This radius is chosen to show the more or less uniform

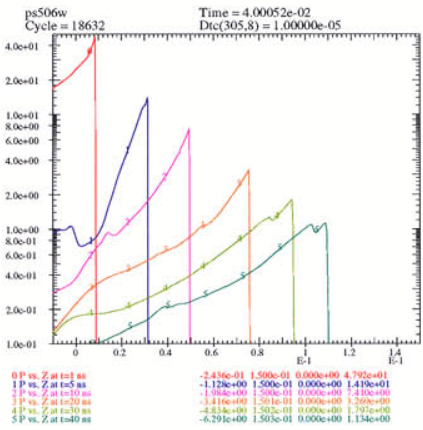

Figure 11: Pressure as a function of $\mathrm{Z}$ coordinate at 6 different times: $t=$ 1, 5, 10, 20, 30, 40 ns.

conditions in the $\mathrm{CH}$ as the shock propagates down the sample. The pressure is shown for the six different times: $t=1,5,10,20,30,40 \mathrm{~ns}$. This plot shows that the shock is not uniformly driven. The sharp peak in the pressure indicates that the rarefaction from the blow-off region has caught up to the shock, even while the laser is still on at $1 \mathrm{~ns}$. After this time, the rarefaction degrades the pressure as it runs down the tube, weakening it from $50 \mathrm{Mb}$ at $t=1 \mathrm{~ns}$ down to roughly $1 \mathrm{Mb}$ at $t=40 \mathrm{~ns}$. As one would expect, the shock speed is also decreasing. Early on the shock velocity is roughly $8 \mathrm{~cm} / \mu \mathrm{sec}$. At late time the shock speed has decreased to $1.4 \mathrm{~cm} / \mu \mathrm{sec}$, down roughly by a factor of 5.5 from early speed.

In Figures 12 and 13 we show the computed side-on radiographs from the CALE simulations. The upper half of each plot shows the radiograph plotted on a linear scale. The values range from 0 to 1 but we have set the range to extend to 1.5 to be completely black (i.e. opaque) so as to see the structure better. The 

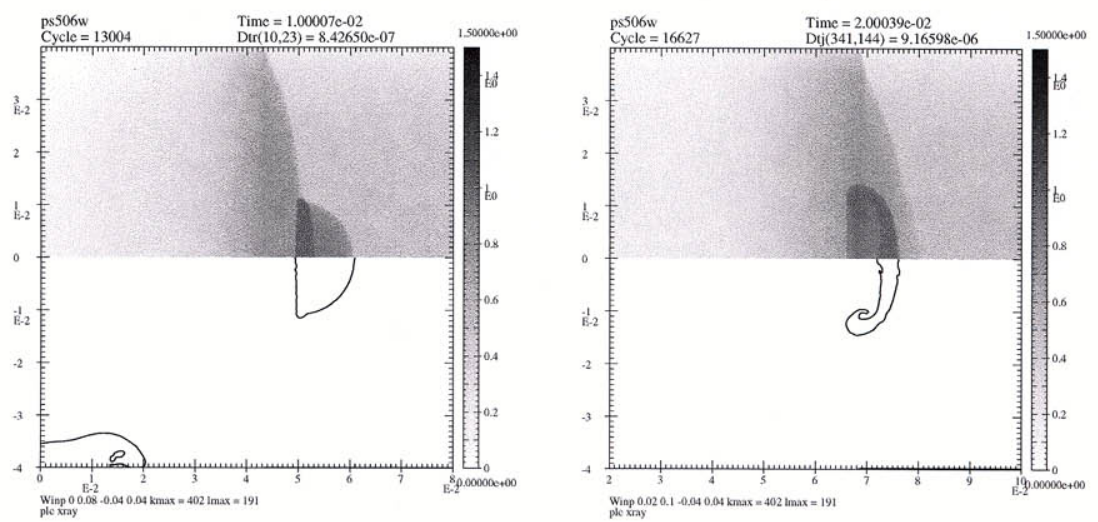

Figure 12: Simulated radiographs from our CALE simulations at (a) $t=10 n s$ and (b) $t=20 n s$. Top half of figure shows the radiograph, while the bottom half simply shows the location of the material boundary.

bottom half of each radiograph simply shows the material boundary. The first figure(Fig. 12a) shows the results at $t=10 \mathrm{~ns}$ with the shock in the exterior foam roughly halfway through the sphere. We can clearly see the shock inside the sphere running ahead of the exterior shock due to the decreased density. An infinite strength shock for a $\gamma=5 / 3$ gas should have a shock speed inside the sphere which is roughly $17 \%$ faster than that outside the sphere for a factor of 2 drop in density inside the sphere. This appears to be consistent with our result. As noted earlier, this is in variance with the experimental picture, where the shock inside the ball appears to be running at roughly the same speed as that outside the ball. Note from the simulated radiograph that the post-shock region in the $\mathrm{CH}$ has a similar intensity to that inside the preshock sphere. The ratio of the opacity in the $\mathrm{CH}$ is roughly $1 / 25$ that inside the sphere. Factoring in the post-shock density (factor of 8 ) and the larger pathlength (factor of 4 at the center), we would predict that the sphere (pre-shock) transmissibility should be within $30 \%$ that of the post-shock $\mathrm{CH}$ foam.

The simulated radiograph at $t=20 \mathrm{~ns}$ (Fig. 12b) shows the shock having emerged from the sphere. The radiograph shows the main body of the sphere compressed in a thin layer with the outer edge showing an rollup from the Richtmeyer-Meshkov and KelvinHelmholtz instabilities. The rollup when projected into cylindrical symmetry yields the "banded" feature leading from the rollup down to the axis of symmetry. As we noted earlier, when comparing with the experiment in Figure 2b, there is little agreement. One could possibly make the case that the main dark feature in this picture is the main rollup, but one cannot explain the material extended further out in radius, nor the material further behind the rollup. The apparent inclination of the axis of symmetry in the sphere to the shock normal is also difficult to explain. 

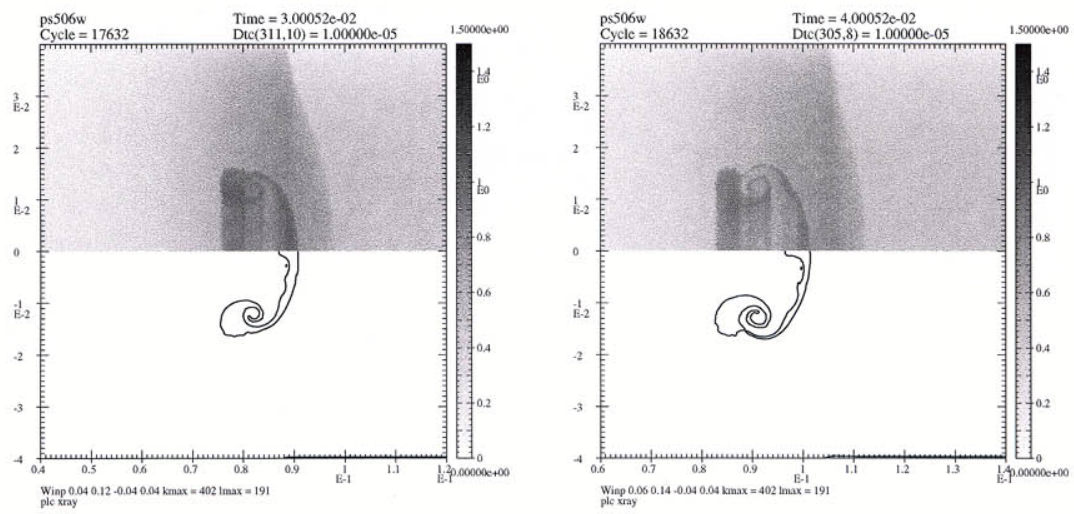

Figure 13: Simulated radiographs from our CALE simulations at (a) $t=30 n s$ and (b) $t=40 n s$. Top half of figure shows the radiograph, while the bottom half simply shows the location of the material boundary.

At $t=30 \mathrm{~ns}$ the simulated radiograph shows the leading edge of the shocked sphere becoming thinner and the rollup at large radii becoming more pronounced. The large thickness of the rollup yields the noticeable streaking when cylindrical symmetry is projected. Comparing with the experiment in Figure 4, the most obvious difference," as we noted earlier, is the two features at large radii (probably rollups) appearing to lead the main body of the sphere. Also in our simulations, the radiographs indicate a compressed leading edge, followed by a lighter, more transparent region, before reaching the more opaque rollup in the trailing parts of the sphere. The experiment seems to show a much more uniform distribution-no well-defined gap is observed, and it has an almost hemispherical shape to it.

At $t=40 \mathrm{~ns}$, the simulated radiograph shows a similar story, that is the leading edge gets thinner, and the gap between the leading edge and the rollup in the Z-direction increases. Most of the mass in the rollup starts to lag even further behind, and yields a simple band structure in the radiograph at the back edge of the sphere. As noted earlier, this radiograph shows much more agreement with the experimental radiograph in Figure 5. The leading shock normal is now coincident with the axis of symmetry, as on would expect. Also, in the simulated radiograph, the leading shock appears to becoming more planar, or at least the curvature is less noticeable. The experiment does finally begin to resemble the simulation. In the experiment there is a nice curvature to the leading edge of the compressed sphere, similar to what is seen in the simulation. There is an almost planar truncation to the trailing edge of the sphere, similar to what is seen in the simulation, unlike what was seen in the experiment at $t=30 \mathrm{~ns}$. There are a couple of important differences though. There appears to be a noticeable darkening in the experimental radiograph along the axis of symmetry which is not seen in our simulations. Also, there appears to be material at large radii at 
the back edge of the sphere in the experimental radiograph which seems to have broken off from the main body of the compressed sphere. We see no such material at large radii in our simulations.

\subsection{Adding Air Gaps}

If one believes the observations at $t=20 \mathrm{~ns}$ and $t=30 \mathrm{~ns}$ as representative, it would seem that one of the deficiencies of the computational models is the inability of the sphere to expand radially. To some extent, this is probably true even if we ignore these two observations and only considered the $t=40 \mathrm{~ns}$ result (a distinct possibility). It certainly appears from

the observations that material extends to large radii, most likely outside the view of the backlighter. After taking a closer look at the engineering drawing of the proposed target, we decided to add true fabrication features. The sphere was placed inside the sample by making a radial slice to open up the $\mathrm{CH}$. Then a region was dug out of one side to place the sphere. The tool used left a cylindrical region with a conical tip towards the irradiated end (see attached figure at end of this report). The sphere was then inserted with glue, and the two pieces of $\mathrm{CH}$ were glued back together. The end result is not quite what we modeled earlier in that there is an air

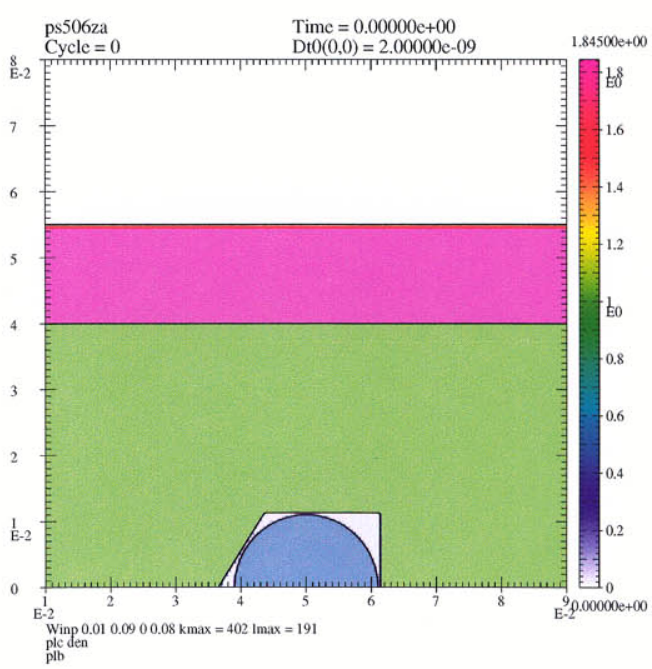

Figure 14: Initial density of the CALE simulations when air gaps are included around the sphere. gap between the sphere and the rest of the $\mathrm{CH}$. The basic premise is that the low density region between the $\mathrm{CH}$ and the sphere will be compressed rapidly after shock passage, causing the material to squirt out radially, possibly dragging parts of the sphere with it.

The resulting initial configuration is shown in Figure 14 where the density is plotted. The evacuated region is nearly to specifications. For this particular run the gap is increased slightly so that there is at least one full cell resolution at the point of near tangency to the sphere. We placed He at $\rho=0.001 \mathrm{~g} / \mathrm{cm}^{3}$ in the gaps, which is roughly 1000 times less dense than the foam, and nearly 500 times less dense than the sphere. This is within a factor of 2 of typical densities of air. As a check, we also performed calculations with higher densities of 

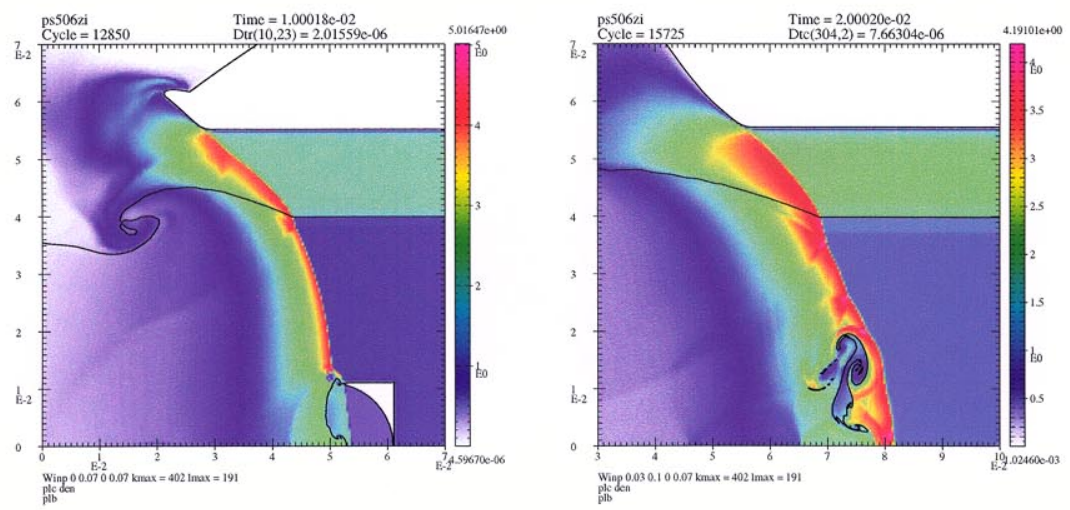

Figure 15: Density at $t=10 \mathrm{~ns}$ and $t=20 \mathrm{~ns}$ when air gaps are included in the calculations per Fig. 14. Dark solid lines represent the material boundaries.

He in the air gaps, up to an order of magnitude higher. We found the results to be similar. Therefore, there seems to be little sensitivity to the exact density chosen in the air gaps.

Figure 15 shows the density at $t=10 \mathrm{~ns}$ and $t=20 \mathrm{~ns}$. Notice that at $t=10 \mathrm{~ns}$, the material boundary of the sphere in the post-shock region near the axis $R=0$ is running faster than the rest of the post-shock sphere. Compare this to Figure 8 where we see a nearly vertical boundary to the post-shock sphere. It is not clear at this time whether this is physical. There is a fairly large air gap near the $R$-axis. The question is does the air gap lead to an acceleration of the post-shock sphere boundary. Obviously, when the shock leaves the $\mathrm{CH}$ and enters the air gap it will speed up due to the low density. For a factor of 1000 density change, we predict that the shock speed will increase by roughly a factor of 2.5 inside the air gap. Thus the shock traveling through different thickness of the air gap will alter the near-planar behavior of the shock. Is this sufficient to explain the behavior of the material boundary - we believe the answer is no. If you look at the location of the shock itself, it is not that much ahead of the material boundary near the axis. This should dictate a much higher density than is observed. We have no explanation for the acceleration of the material boundary near the axis. Therefore we believe it to be a numerical artifact. If the effect is real, we should also see a similar behavior at large radii where there is another large air gap. We admit there is some evidence of such curvature at large radii. That is, it appears that the slowest part of the post-shock material boundary is near the tangent point of the sphere, where there is practically no air gap at all. However, the effect near the $R$-axis is significantly larger, with the material boundary being only $0.001 \mathrm{~cm}$ behind the shock front.

At $t=20 \mathrm{~ns}$ the shock has run all the way through the sphere. It appears that the compressed sphere has broken off from the $R$-axis. No doubt this is a consequence of what 
looks like the a jet-type feature forming along the $R$ - axis. As mentioned above, it is my opinion right now that this is a numerical phenomena, rather than physical one..

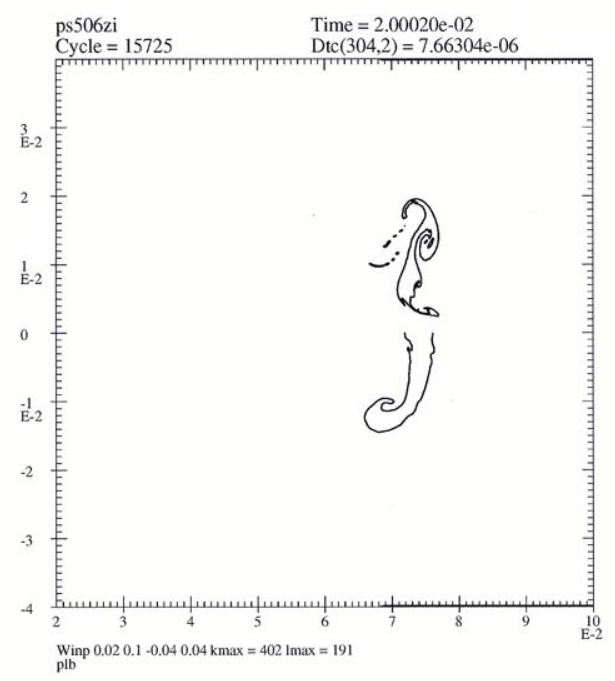

Figure 16: Material boundary at $t=20 \mathrm{~ns}$. Bottom part $(R<0)$ is from the earlier standard calculation in Figure 8. Top part $(R>0)$ is for the current calculation which includes pockets of low density air around the sphere (see Fig. 14).
Notice the rollup at large radii is much more developed and actually tilted in the forward direction, i.e. towards the emergent shock. To show the comparison with our earlier calculation, we show in Figure 16 a plot of just the material boundary at $t=20 \mathrm{~ns}$. The upper half of the plot is from the current calculation, while the lower half is from the standard calculation of Figure 8. If we ignore the "jet" along the axis in the present calculation, the most striking difference is the rollup at large radii. In the previous calculation, the rollup is slow to form and it trails the main body of the compressed sphere. Whereas, in the current calculation the rollup is much more developed and is leading the main

body of the compressed sphere. The other difference is that the rollup has expanded outward in the radial direction more in the current calculation. The outer edge of the rollup is roughly at $R=0.02 \mathrm{~cm}$, as opposed to $R \approx 0.015 \mathrm{~cm}$ in the previous calculation. Both of these trends are in the right direction in order to provide better agreement with the observations. Although, as we discuss later, the details are far from in agreement.

So what has led to this behavior in the rollup? Apparently it is due to the large evacuated region near the upper right side of the initial sphere (see Figure 14). Once the shock emerges from the sphere, it speeds up dramatically in the under-dense region. The increased shock speed provides for a faster post-shock speed and a lower post-shock pressure. Both of these facts allow the outer parts of the compressed sphere to expand more radially and to the right. This allows the rollup to actually lead the rest of the compressed sphere and start to tilt the rollup to the right.

Figure 17 shows the density at $t=30 \mathrm{~ns}$ and $t=40 \mathrm{~ns}$. At $t=30 \mathrm{~ns}$ the rollup has continued and expanded radially out to roughly $R=0.025 \mathrm{~cm}$. This is significantly larger than the previous result, where the outer edge of the rollup was only at $R=0.017 \mathrm{~cm}$ at 

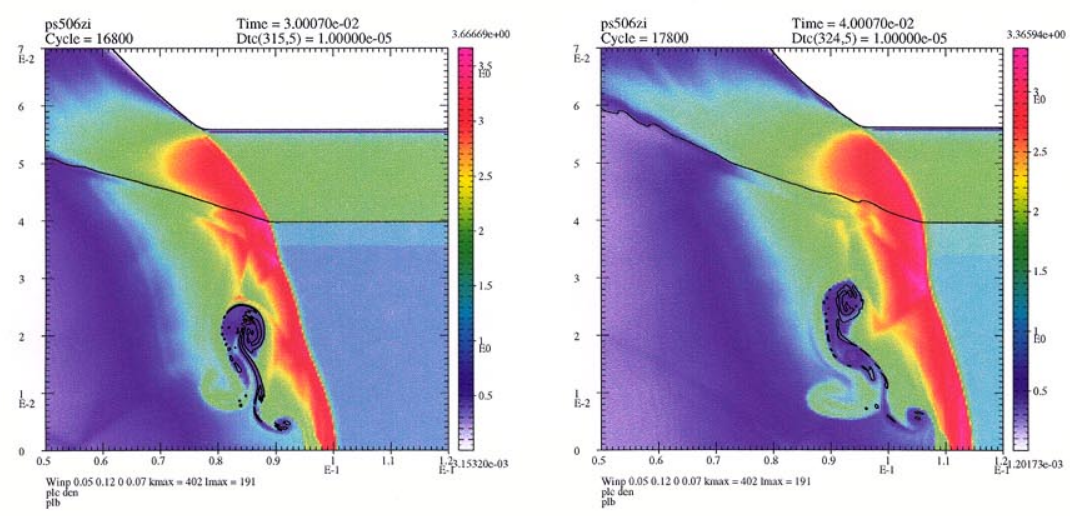

Figure 17: Density at $t=30 \mathrm{~ns}$ and $t=40 \mathrm{~ns}$ when air gaps are included in the calculations per Fig. 14. Dark solid lines represent the material boundaries.

this time (Fig. 9). The rollup is much more in line vertically (i.e. at constant $Z$ ) than that in Figure 9. The rollup is also further downstream, with no part less than $Z=0.081 \mathrm{~cm}$. While in Figure 9 we see that the rollup lags well behind the main body of the compressed sphere, reaching as far back as $Z=0.075$. There is still evidence of a jet near the $Z$-axis, which expands out in radius now to almost $R=0.005 \mathrm{~cm}$.

Finally, at $t=40 \mathrm{~ns}$, much of the structure has broken up into little islands. The only noticeably connected region is the rollup. It has now expanded radially out to nearly $R \approx 0.03 \mathrm{~cm}$. The rollup is slowing down in the Z-direction compared with the parts of the sphere at smaller radii. The jet near the Z-axis is well formed with a nice rollup forming at $R \approx 0.005, Z \approx 0.105$. For ease of comparison we show in Figure 18 the material boundaries at $t=40 \mathrm{~ns}$. As in Figure 16, $Z<0$ shows the results from the earlier calculation shown (Fig. 9), while for $Z>0$ the material boundaries are drawn for the present calculation.

Figure 18 shows the rapid breakup of the sphere into small islands. This indicates that there must be a lot more mixing going on in these models due to the pockets of air initially around the sphere. If one ignores the jet along the axis, then tracing a line along the outer edge of these islands of material, we find that it does have some similarity to the observation at $t=40 \mathrm{~ns}$. Namely, there is a nice simple spherical curvature out to $R \approx 0.015 \mathrm{~cm}$, whereupon the curvature changes direction near the outer rollup. Although this result does not agree in detail with the observations, it presents a picture closer to that of the observations. Without the air pockets, the compressed sphere is much smoother; the rollup is less well developed and lagging well behind the central part of the compressed sphere, extending back roughly $200 \mu \mathrm{m}$ from the leading edge. Also the rollup, in the case without air pockets, does not appear to expand enough radially. 

of

Figure 19 shows the simulated radiographs at $t=10$ and $t=20 \mathrm{~ns}$ on the upper half

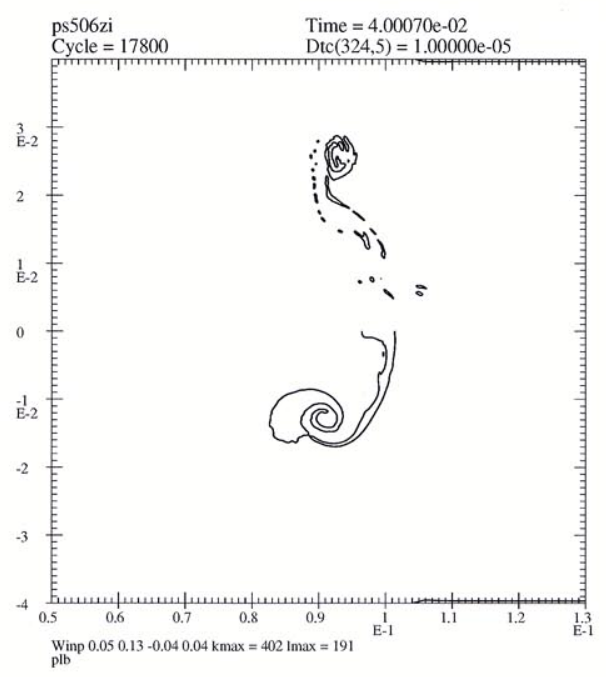

Figure 18: Material boundary at $t=40 \mathrm{~ns}$. Bottom part $(R<0)$ is from the earlier standard calculation in Figure 8. Top part $(R>0)$ is for the current calculation which includes pockets of low density air around the sphere (see Fig. 14). the plot, and the location of the material boundaries on the lower half. As was the case earlier, we plot the simulated radiographs on the scale 0 to 1.5 for better contrast. At $t=$ $10 \mathrm{~ns}$ the radiograph appears very similar to the earlier model without the air pockets (Fig. 12). The compressed layer in the radiograph appears smooth, i.e. there is no evidence of the jet along the axis, even though the material boundary obviously displays it. It must be that the outer radii of the compressed sphere are sufficiently thick to mask the evacuated center in the radiograph.

At $t=20 n s$ (Fig. 19b) there is, however, a discernible difference in the simulated radiograph compared with Figure 12. At large radii we see evidence of the rollup tilted forward towards the transmitted shock. This rollup is of sufficient mass that, when projected over $360^{\circ}$ in forming the radiograph, produces a radial band structure all the way down to the origin, even when the material boundary indicates little opaque material near the Z-axis. That is, projection effects of the rollup at large radii are masking the details at small radii. The main body of the compressed sphere is between $R=0.005-0.010 \mathrm{~cm}$, the projection of which produces another smooth radial band at $Z \approx 0.073 \mathrm{~cm}$ out to $R=0.01 \mathrm{~cm}$. In the trailing parts little islands of sphere material have broken off producing noticeable streaks at $Z<0.07 \mathrm{~cm}$. Overall, the radiograph is more complicated than that in Figure 12, which simply shows a compressed, curved shell with a rollup at the end. We admit that Fig. 19b still does not agree in detail with the observation in Figure 3. But it does have some desirable characteristics, including expansion to larger radii, and the rollup leading the main body of the compressed sphere- both of which were lacking in Figure $12 \mathrm{~b}$.

Figure 20 shows the simulated radiographs for $t=30 \mathrm{~ns}$ and $t=40 \mathrm{~ns}$. At $t=30 \mathrm{~ns}$ the compressed regions of the sphere have become noticeably thinner, both in the rollup and in the main body. In addition, more islands of material have broken off. This leads to streaks in the simulated radiograph. The islands of material in the jet, near the Z-axis, are 

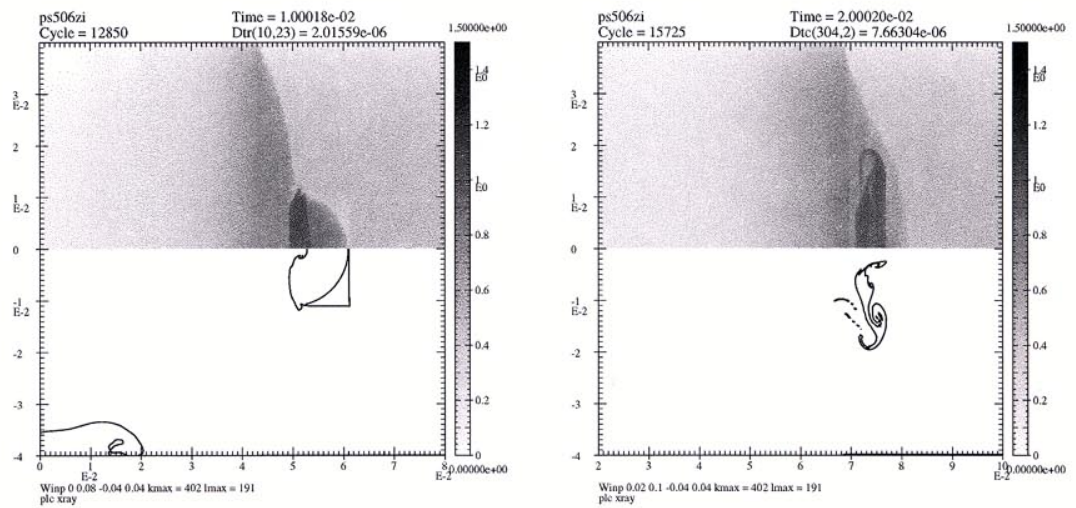

Figure 19: Simulated radiographs from our CALE simulations including air gapssat (a) $t=10 \mathrm{~ns}$ and (b) $t=20 \mathrm{~ns}$. Top half of figure shows the radiograph, while the bottom half simply shows the location of the material boundary.
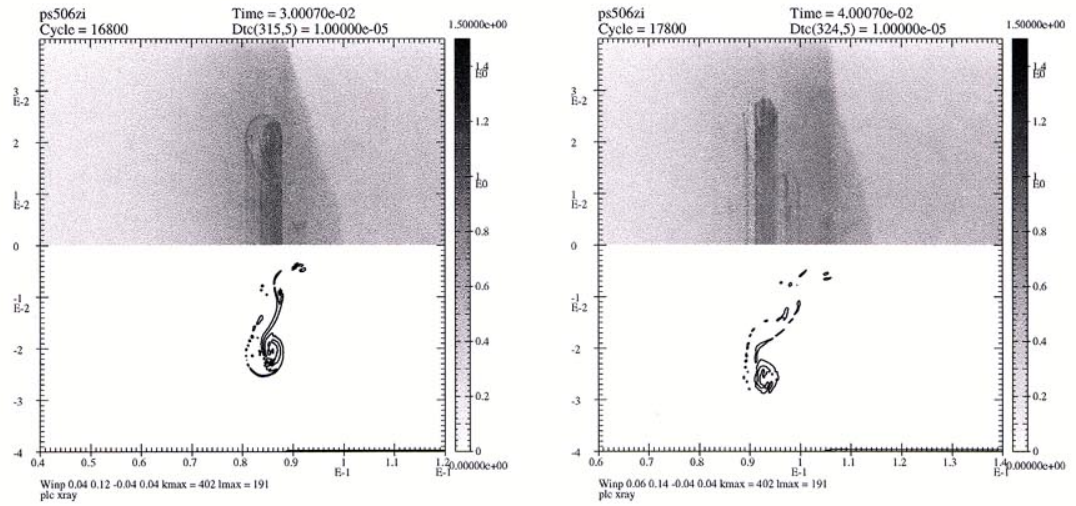

Figure 20: Simulated radiographs from our CALE simulations including air gaps at (a) $t=30 \mathrm{~ns}$ and (b) $t=40 \mathrm{~ns}$. Top half of figure shows the radiograph, while the bottom half simply shows the location of the material boundary. 
hardly visible in the radiograph. It is clear that the velocity of the rollup in the Z-direction is slowing, since it is now slightly behind the main body of the compressed sphere at smaller radii. Overall, it is difficult to conclude whether or not this simulated radiograph provides improved agreement with the observation over that in Figure 13a, since really neither one looks a whole lot like the observation. The observations seem to indicate rollups at large radii which have broken off and are leading the main body of the sphere. Also the rest of the sphere has a nicely rounded shape to the trailing edge of the sphere, neither of which are seen in either Figure 13a or Figure 20a.

At $t=40 \mathrm{~ns}$ the main body of the compressed sphere has broken up into a number of little islands, both on the leading and trailing edges. The only continuous structure is the rollup near $R=0.03 \mathrm{~cm}$. All of the broken islands produce streaks in the simulated radiograph. Obviously, this radiograph does not really resemble the observed radiograph in Figure 5. However, it does have the characteristic that the curvature of the main body of the compressed sphere is of the right type. This is best seen by looking at the material boundary plot. Notice also the change in slope at large radii, approaching the rollup; this also appears to be a feature of the observation (Fig. 5). Unfortunately, by breaking up into so many small islands, the shape is somewhat lost in the simulated radiograph. With such a large breakup, one should probably be somewhat skeptical of the simulated results. The existence of the jet-like behavior near the $Z$-axis should also give one some pause. However, even with these problems, the shape of the leading edge of the material does seem more suggestive of the observations than did the earlier simulations (Fig. 13b). Although we admit that in the new calculations the curvature of the material boundary occurs at too large a radii.

\section{Conclusions}

We have compared CALE simulations with an experiment on Omega of a shock passing through a medium which has an under-dense sphere. We have noted, without any detailed comparison with simulations, that the observations seem to present a number of difficulties; not the least of which is that it appears the radiographs do not present a logical sequence of events. This is particularly true when analyzing the late time radiographs at $t=40 \mathrm{~ns}$ with the earlier $t=20 \mathrm{~ns}$ and $t=30 \mathrm{~ns}$. It is difficult to explain how a model progresses from one of somewhat chaotic behavior $(t=20 \mathrm{~ns}$ and $t=30 \mathrm{~ns})$, toward one of more uniformity $(t=40 \mathrm{~ns})$. Other problems include an apparent angle between the axis of symmetry of the event and the shock normal. Not only does this angle appear, it further disappears by late time $(t=40 \mathrm{~ns})$. Another problem with the observations is the presence of rollups breaking off and leading the main body of the compressed sphere $(t=30 \mathrm{~ns})$. Although, as noted in the last section, the inclusion of pockets of air between the under-dense sphere and the ambient foam may contribute to such behavior. It seems clear that since only a single snapshot is obtained from each shot, that manufacturing or laser drive variability may have played some role in the observed behavior.

We have performed CALE simulations of an underdense sphere simply immersed inside the Polystyrene foam. The results are fairly simple to describe. The sphere is first compressed 
into a layer with the outer part (large radii) forming a rollup due to Richtmeyer-Meshkov instability. After the shock passes, the central compressed part simply advects downstream, while the rollup begins to slow down due to interaction with ambient post-shock medium. This leads to a general thinning of the entire layer as it is stretched out due to the slowing of the rollup relative to the central compressed region.

We have performed another set of calculations where we include in more detail the way in which the under-dense sphere is inserted into the sample. This involves drilling out a conical section, then glueing the sphere inside the evacuated region. Since the shape of the evacuated region does not match the sphere, there are air pockets between the sphere and the ambient $\mathrm{CH}$ foam. The hope was that this detail was unimportant, unfortunately we find this not to be the case. We performed a calculation where we accurately model the evacuated region and inserted He at $\rho=10^{-3} \mathrm{~g} / \mathrm{cm}^{3}$ (roughly appropriate for air). We have computed several other models with densities up to an order of magnitude larger and see little difference in the results. Thus the results seem not to be sensitive to the exact density of material in the gaps between the foam and the sphere. The results differ from the simply embedded sphere in two principle ways: (1) there is much more radial expansion, reaching nearly $50 \%$ larger radii at $t=40 \mathrm{~ns}$; and (2) the rollup at large radii, early on, leads the central part of the compressed sphere, rather than lagging behind. The latter feature is transitory, eventually the rollup slows down and by $t=40 \mathrm{~ns}$ lags the central compressed region. There are problems with these calculations though. First of all, a jet seems to form along the $Z$-axis. Even though there is a pocket of air between the foam and the sphere along the Z-axis, we see no reason why this should lead to the formation of a noticeable jet. At this point we tend to believe this to be a numerical artifact. The second problem, is that there is considerably more breakup of the compressed sphere into small pockets or islands. Our belief in the ALE algorithms is somewhat tested when the mixing is sufficient to produce a breakup into a large number of small islands.

In summary, it appears that more shots are required to determine the veracity of the observations, particularly at the intermediate times of $t=20 \mathrm{~ns}$ and $t=30 \mathrm{~ns}$. Unfortunately, from the calculations, it appears that the exact details of how the sphere is embedded inside the sphere does matter to the evolution. Therefore care must be used to include this effect in the simulations, and care must be used to guarantee the consistency of target manufacturing. 


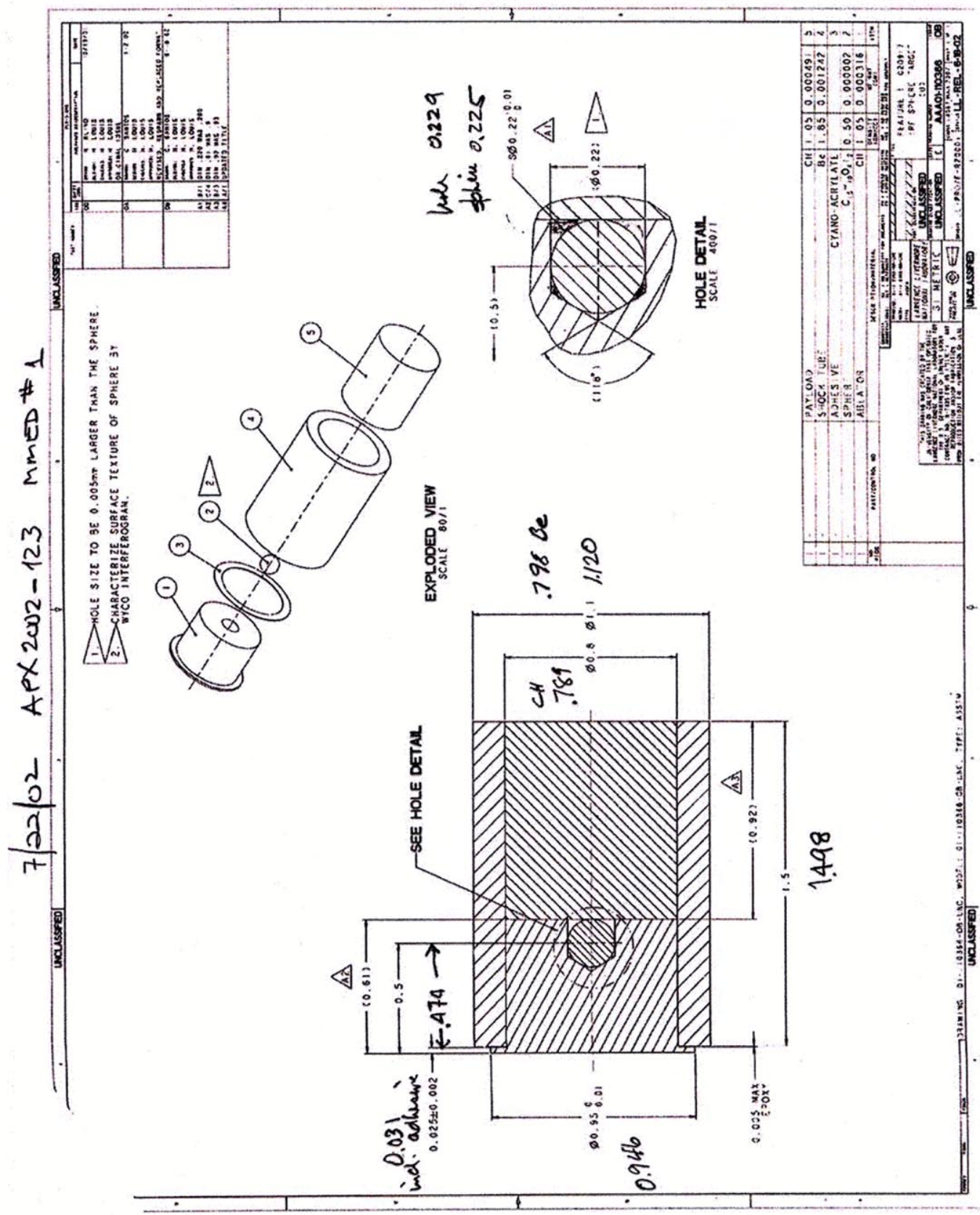




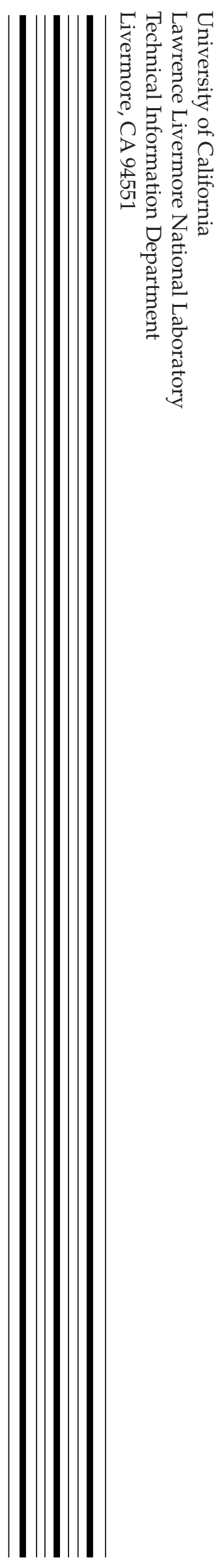

